\title{
Tangence
}

\section{À côté de cette porte qu'est la mort ( La porte à côté , de Paul Zumthor}

\section{Guy Lavoie}

Numéro 49, décembre 1995

Les littératures francophones de l'Afrique et des Antilles

URI : https://id.erudit.org/iderudit/025883ar

DOI : https://doi.org/10.7202/025883ar

Aller au sommaire du numéro

Éditeur(s)

Tangence

ISSN

0226-9554 (imprimé)

1710-0305 (numérique)

Découvrir la revue

Citer ce compte rendu

Lavoie, G. (1995). Compte rendu de [À côté de cette porte qu'est la mort ( $L a$ porte à côté, de Paul Zumthor]. Tangence, (49), 121-125.

https://doi.org/10.7202/025883ar d'utilisation que vous pouvez consulter en ligne.

https://apropos.erudit.org/fr/usagers/politique-dutilisation/ 


\title{
À côté de cette porte qu'est la mort \\ Paul Zumthor, La porte à côté, Montréal, l'Hexagone, 1994, $189 \mathrm{p}$.
}

\begin{abstract}
Derrière moi, beaucoup d'autres sont morts. Sans trop y songer, soufflant sur leur cendre, j'ai rallumé en moi leur feu. Il faut qu'il me brûle. Nul ne le saura, tandis que je poursuivrai cette marche de toujours dans le labyrinthe, sans fil d'Ariane, vers la chambre de verdure où la Belle au Bois a fermé les yeux. (p. 120-121)
\end{abstract}

Je m'assieds enfin dans la quiétude de mon salon pour lire le dernier recueil de nouvelles de Paul Zumthor, La porte à côté, que je tiens comme un trésor depuis que j'ai quitté la librairie. J'ai hâte de l'ouvrir pour en libérer les esprits magiques dont nous parlait Jorge Luis Borge ${ }^{1}$ en convoquant Emerson. Bien malgré moi, ma lecture ne peut cependant être que biaisée, influencée par le décès de l'auteur que j'ai appris quelques mois plus tôt, par hasard. Même si la "science textuelle" m'a appris à dissocier l'œuvre de la référence biographique, je ne puis m'empêcher d'appréhender ce livre comme l'aboutissement d'un parcours ${ }^{2}$ avec tout ce que cela implique d'héritage. C'est un peu comme si je m'attendais à ce que la narration de cette ceuvre de fiction soit "accompagnée de ces voix qui, comme le souligne Chateaubriand, ont quelque chose de sacré, parce qu'elles sortent du sépulcre" ${ }^{3}$, ces voix qui sont traversées par l'angoisse du temps qui passe et de la mort.

Avant même de tourner la page couverture, je me heurte à cette porte de bois aux motifs géométriques qui, agencés, laissent deviner des sabliers à cette porte placée en arrière de colonnes

1 Jorge Luis Borge, Conférences, Paris, Gallimard, coll. "Folio/essais ", 1985, p. 91.

2 D'après la bibliographie fournie par l'éditeur, cet auteur a publié, depuis 1938, plus d'une trentaine d'œuvres de fiction et d'ouvrages critiques.

3 François René de Chateaubriand, Mémoires d'outre-tombe, tome I, Paris, Flammarion, 1940 , p. 8. 
122

d'or qui semble fermée sur l'au-delà. Dès les premières phrases de ce qui ressemble à une préface, Paul Zumthor parle de cette porte qu'il ne peut ouvrir, devant laquelle il demeure inlassablement, puis de la mort qu'il coudoie depuis l'aube de sa vie: "Le temps s'écoule ainsi: le temps d'une vie, je veux dire de cet interminable monologue que nous tenons, pour combler notre lieu clos, face à la Mort. Nous voudrions bien que ce fût un dialogue, mais la Mort ne répond pas." (p. 7) Ce monologue se poursuit ensuite dans la presque totalité des dix-huit nouvelles de ce recueil qui se divise en trois parties respectivement intitulées: "Récits", "Esquisses" et "Médiévales". Par moments, il devient plus insistant, se transforme en un questionnement existentiel: "Peut-être est-on déjà mort" (p. 107), et quelques pages plus loin: "Peut-être ne mourrai-je pas. La mort est trop cruelle. La mort n'appartient pas même à celui qui meurt, mais aux survivants à qui elle offre son spectacle." (p. 155)

Mais cette mort qui se donne en spectacle sous le regard et dans l'esprit n'est qu'une façade qui dissimule, en réalité, une volonté d'exhiber la vraie vie, celle qui est composée de ces instants magiques où "le monde devient parfait" (p. 111), de ces événements à la fois insignifiants et sordides, quoique humains, de ces détails qui, trop souvent, échappent à notre attention, nous qui sommes happés par la vitesse et l'urgence de mener nos vies. En effet, l'écriture dans La porte d'à côté est porteuse de cette nécessité d'explorer ce que Milan Kundera appelait avec justesse "la vie humaine dans le piège qu'est devenu le monde " 4 . Même s'il reste coincé derrière la porte, cet auteur jette souvent un oil à la fen-être et, à chaque nouvelle, nous fait découvrir un personnage différent autour duquel il esquisse une intrigue, un être de chair et de sang en qui il nous est facile de croire, un être humain sur lequel pèse l'ombre de petits soucis, peut-être de ces "grandes souffrances cachées" ( $p$. 92). Il tente de pénétrer ces êtres souvent solitaires qui se dérobent continuellement, lui qui ne peut saisir que les apparences.

À première vue, ce qui émerge de cette ouvre, c'est moins ce que dit le texte que ce qu'il ne dit pas, que ce qui apparaît en filigrane au détour d'une phrase, dans l'espace vide entre deux mots, entre deux nouvelles. Comme l'auteur l'affirme lui-même, "il

4 Milan Kundera, L'art du roman, Paris, Gallimard, coll. "Folio", 1995, p. 39. 
ne reste que le poids actuel de ce qu'on dit; le poids plus lourd encore de ce qu'on fait. (p. 92). Et, à la suite, de la disparition du personnage, nous nous retrouvons seuls, avec "le désarroi qu'a engendré sa brève présence" (p. 47) et l'incertitude qui "embrüme alentour de nous les choses, comme si elle rayonnait de notre cœur même, peuplée d'images fragmentaires, pas nées, remontant en nous d'une époque où l'être hésitait encore à vivre" (p. 48). Force est de constater, par ailleurs, que le faisceau que projette Paul Zumthor - qui implique donc une certaine incomplétude trouve son origine dans les motivations de ces poètes populaires qu'il décrit dans "Interview": "bricoler avec ces rebuts [ses personnages le font souvent: le vieux poète, "La dame au buffet", le "Docteur Schneider"] un espace imaginaire où l'on pourrait vivre" (p. 12), où l'on finirait par oublier l'en-dehors ( $\mathrm{p} ; 7)$.

C'est probablement dans le récit "L'inconnue" - nouvelle présentée en partie sous la forme d'un journal - que l'auteur rend plus lisible et visible le jeu qu'il opère avec les éléments de sa création. Dans cette fiction, l'instance narrative, un écrivain, consigne pendant un an dans un journal personnel tous les faits et gestes d'une femme qui demeure dans l'immeuble d'en face. Par la fenêtre où il a poussé sa table de travail (ce que la femme va faire à son tour quelques mois plus tard), il regarde, sans cesse, toujours avec un peu plus d'intérêt, cette inconnue, glisse dans une sorte de voyeurisme à demi assumé. Certes, "il se reproche cette curiosité, et pourtant s'en défend mal, puis se pose des questions sur ce qu'il a vu. Il s'applique à en faire un amusement" (p. 54). Il pourrait bien connaître son nom qui "figure sans doute sur la porte d'entrée de l'immeuble ou sur l'une des boîtes aux lettres" (p. 53) afin de la rencontrer, de lui parler, bref de rompre le silence, mais il s'y refuse. Pourquoi une telle pudeur, un tel repli sur soi? "Pourquoi, je ne sais, répond-il, elle sans doute ne le sait davantage. Simplement impossible» (p. 55).

À bien y regarder, cette ignorance volontairement entretenue d'où découle une "souveraine liberté" (p. 57) constitue l'un des principaux ressorts de l'intrigue. De fait, le regard contemplatif de l'instance narratrice, semblable en plusieurs points à celui du chat de la femme ${ }^{5}$, gravite autour de deux mots clés: intérêt et

5 Le comportement de l'animal dédouble souvent celui du narrateur-écrivain: "Il ne cesse de manifester sa présence: il passe des heures à dormir ou à rêvasser, allongé sur l'appui de la fenêtre. Il règne sur les lieux". (p. 53) 
124

soupçon (p. 54). L'équation est simple: plus l'inconnue restera étrangère, plus grands seront les soupçons, l'intérêt à percer son mystère. Ce n'est pas pour rien que le protagoniste censure son discours à tout bout de champ, refoule toute idée trop réelle, "préférant supposer quelque troublant mystère" (p. 54). Ce recueil "n'examine pas la réalité mais l'existence. Et l'existence n'est pas ce qui s'est passé, l'existence est le champ des possibilités humaines, tout ce que l'homme peut devenir, tout ce dont il est capable." 6 La dynamique de la narration de La porte à côté repose sur le pouvoir cathartique de nous rendre non seulement témoins mais aussi complices du regard pervers de ce voyeur. Toutefois, sa curiosité n'est pas malsaine ou impudique, elle est plutôt celle de quelqu'un dont le seul but est de comprendre, oui, comprendre ce qu'est l'aventure humaine.

Cette fen-être derrière laquelle se dissimule l'homme, cet espace vitré, cet objet humain jouant à la fois un rôle interprétatif et critique, permet non seulement de voir l'inconnue, mais renvoie aussi à l'image de celui qui contemple. Tout au long de cette nouvelle, voire de ce recueil, Paul Zumthor, fait davantage qu'embrasser du regard l'autre; il dévoile, souvent avec humour, dans un style bien vivant, bon nombre d'images fragmentées de ses pensées, de sa vaste expérience du monde. Autobiographie? Les nombreuses mises en abyme, la référence intertextuelle à certains de ses ouvrages ${ }^{7}$, les discussions sur le genre autobiographique "aux buts secrets (nécessairements troubles)" (p. 95), les similitudes entre les professions de l'auteur et celles des protagonistes (écrivain, professeur, littéraire), la réflexion sur l'écriture (entre autres dans "Graffiti"), tout pourrait le laisser croire, mais cet auteur écarte du revers de la main cette hypothèse, tenant à rester fidèle aux propositions esthétiques de l'exorde: montrer de "belles images, plus émouvantes et vraies que le vrai" (p. 7). De plus, ne dit-il pas: "Mais le passé n'est pas un présent dépassé: c'est quelque chose de perdu, que jamais l'on ne posséda, et qui peut-être n'exista point" (p. 111-112)?

6 Milan Kundera, op. cit., p. 57.

7 Dans la nouvelle "La rose", nous retrouvons, entre autres, la phrase suivante: "Cette chapelle blanche, le tombeau trop pur entre ses grêles colonnes: première halte devenue pour nous rituelle depuis que j'ai publié mon livre sur Abélard et Héloise" (p. 40). Cette référence trouve écho avec les ouvrages Lettres de Héloise et Abélard (traduites) et Abélard: Lamentations, écrites par l'auteur. 
Tout comme l'homme en train de lire "Dans le métro", un instant, ma main referme ce livre, laissant un doigt entre les pages. Mon regard vogue au loin; plus rien n'existe autour de moi (p. 104). Du palimpseste de ma mémoire surgit alors une phrase de Danièle Sallenave qui s'impose à mon esprit: "La littérature nous fait aborder les rives mystérieuses, [...] où la mort est vaincue. $" 8$

Guy Lavoie

8 Danièle Sallenave, Le don des morts. Sur la littérature, Paris, Gallimard, 1991 , p. 113. 